\title{
Influence of slow laser modulations on the amplification process of beat-wave accelerators
}

\author{
F. B. Rizzato \\ Instituto de Física, Universidade Federal do Rio Grande do Sul, Caixa Postal 15051, 91500 Porto Alegre, \\ Rio Grande do Sul, Brazil
}

(Received 17 January 1991; revised manuscript received 10 May 1991)

\begin{abstract}
In this work we analyze how amplitude modulations of the laser field affect the plasma-wave amplification in the beat-wave accelerator. It is shown that under certain resonance conditions, modulated lasers may cause the plasma wave to grow beyond the limits calculated from homogeneous models with the same initial-energy content.
\end{abstract}

In the beat-wave accelerator, an intense plasma wave with frequency very close to the plasma frequency $\omega_{p}$ is generated by the beating of two high-frequency electromagnetic modes with frequencies $\omega_{1}$ and $\omega_{2}$ such that $\omega_{1}-\omega_{2}-\omega_{p} \equiv \delta\left(\ll \omega_{p}\right)[1-4]$. In most of the models for this kind of process, the amplitude of the light waves is assumed to be constant. If one thinks that the lasers are being constantly fed by some external source, this could be a justifiable approximation, but even in those energyconstant cases other processes may induce some inhomogeneity in their amplitudes. In this regard, besides laboratory-controlled amplitude modulations, which will be shown to be of some interest, one should consider the modulational instability (MI) of the laser and electrostatic fields [5-7], where it is seen that the relativistic correction of the electronic mass as well as ponderomotive effects produce nonlinear changes in the group velocity of the waves, causing some energy redistribution over the entire space, without any variation of the total content. Taking those observations into consideration, assuming propagation along the $x$ axis, introducing the normalized density fluctuation of the electrons in the field of the plasma wave as $n / n_{0}=a_{p} e^{\left(i k_{p} x-\omega_{p} t\right)}+$ c.c., defining the normalized inhomogeneous electromagnetic pumps as

$$
e a_{1,2}^{\text {pump }} / m c^{2}=a_{1,2}(x, t) e^{\left(i k_{1,2} x-\omega_{1,2} t\right)}+\text { c.c. },
$$

and assuming $\partial_{t} a_{p, 1,2} \ll \omega_{p} a_{p, 1,2}, \partial_{x} a_{p, 1,2} \ll k_{p} a_{p, 1,2}$, one can derive an evolution equation for the induced electrostatic wave in the form

$$
i\left(\partial_{t}+v_{g l} \partial_{x}\right) a_{p}=-\delta a_{p}-\left|a_{p}\right|^{2} a_{p}+S(x, t),
$$

where we are considering $S(x, t)=\frac{2}{3}\left(c k_{p} / \omega_{p}\right)^{4} a_{1} a_{2}$ with $t \rightarrow \frac{3}{4}\left(\omega_{p} / c k_{p}\right)^{2} \omega_{p} t, x \rightarrow \frac{3}{4}\left(\omega_{p} / c k_{p}\right)^{2}\left(\omega_{p} / c\right), \delta \rightarrow$ $\frac{4}{3}\left(c k_{p} / \omega_{p}\right)^{2} \delta / \omega_{p}$, and $v_{g l}$ (the group velocity of the plasma waves) $\rightarrow v_{g l} / c$. The cubic term in the amplitude of the plasma wave originates from relativistic corrections to the electronic mass and is fundamental in determining the saturation level of the process.

When the source function $S$ is space-time independent $\left(S_{0}\right)$, one can perform yet another normalization to write the evolution equation in the form

$$
i\left(\partial_{t}+v_{g l} \partial_{x}\right) a_{p}=-\delta a_{p}-\left|a_{p}\right|^{2} a_{p}+1
$$

with $a_{p} \rightarrow a_{p} / S_{0}^{1 / 3}, \delta \rightarrow \delta / S_{0}^{2 / 3}$, and $(x, t) \rightarrow(x, t) S_{0}^{2 / 3}$.

Our next step is to perform the following coordinate and field transformations:

$$
t \rightarrow t, \quad x \rightarrow \xi=x-v_{g l} t, \quad a_{p}=a e^{i \theta},
$$

in order to obtain the following two coupled equations for the phase and amplitude of the plasma wave:

$$
\partial_{t} a=-\sin \theta
$$

and

$$
a \partial_{t} \theta=-\cos \theta+\delta a+a^{3} .
$$

From these two equations it is readily shown that

$$
\partial_{t}\left(a \cos \theta-\frac{\delta}{2} a^{2}-\frac{a^{4}}{4}\right)=0,
$$

from which we conclude that the quantity in large parentheses is a conserved (in $t$ ) one, which from now on will be denoted as $H$.

Now we are able to calculate the maximum amplitude obtainable in the process when the initial state is the one with $a=0$ (or $H=0$ ). To do that, it suffices to note that when $a$ is maximum Eq. (4) indicates $\cos \theta=$ \pm 1 , a result that when inserted in the above-mentioned conserved quantity furnishes the various possibilities of solutions (roots) represented in Fig. 1, where we indicate the $\delta=-3$ situation. From this figure it is possible to arrive at two conclusions.

(i) If $\delta$ is larger than a certain critical value, only one maximum is present.

(ii) If $\delta$ is smaller than this critical value three maxima are possible, two of which grow with decreasing $\delta$.

Those considerations could lead us to think that in order to have higher plasma-wave amplitudes, it would be enough to work with lasers with very large (and negative) frequency mismatches. However, it has been shown that this is not so. Based on effective (or Sagdeev) potential considerations it is possible to see that, for each value of $\delta$, the plasma-wave amplitude oscillates with its nonlinear frequency only in the allowed (A) regions of Fig. (1), the other regions representing impenetrable "potential barriers" [8]. As already noted [8] even a mismatch that is 


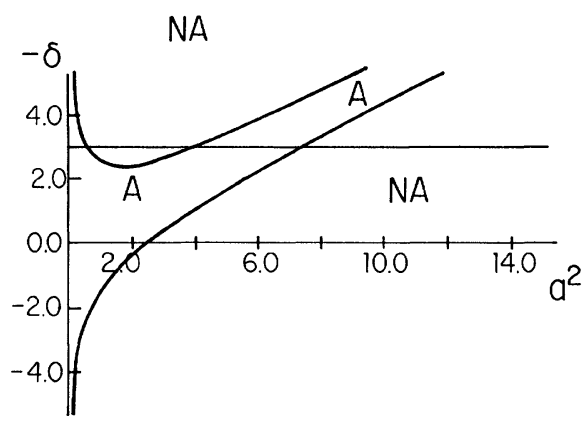

FIG. 1. Maximum amplitudes of the plasma waves. The symbol A (NA) denotes allowed (not allowed) regions of nonlinear oscillations. All the quantities represented in the figures are dimensionless.

close to and slightly larger than the critical one offers no practical advantage; for in this case, although the large root is accessible (it is, in fact, the only one present), the long time consumed close to the (virtual) smallest ones would be incompatible with beam loading, which must occur in much shorter time scales.

That is what could be said about homogeneous pump lasers. Next we focus our attention on the case of laser fields modulated in space and time, where we shall see that under certain circumstances, the electrostatic beat wave could be noticeably enhanced. Actually, we shall see that when the modulation is in resonance with the nonlinear oscillatory frequency of the plasma-wave envelope and the frequency mismatch is below the critical one, there can occur some induced sort of "jump" between small and large amplitudes. This jump is caused by resonant energy transfer between the modulations of the electromagnetic field and the nonlinear oscillations of the electrostatic mode envelope, with a subsequent destruction of the "potential barrier" that separates those two types of roots. In order to pursue our topic, let us assume that one of the laser fields is modulated. Considering a simple model for the modulation (which could be artificially obtained in the laboratory, anyway), we may write

$$
S(x, t)=1+\epsilon e^{i(K x-\Omega t)},
$$

where $\epsilon(0 \leq \epsilon \leq 1)$ is a quantity that measures the degree of modulation.

The exponential factor for this field may be transformed accordingly to the rules defined by Eqs. (3):

$$
e^{i(K x-\Omega t)}=e^{i K \xi} e^{i\left(K v_{g l}-\Omega\right) t},
$$

which is a fact that enables us to write our equation in the form

$$
i \partial_{t} a_{p}=-\delta a_{p}-\left|a_{p}\right|^{2} a_{p}+1+\epsilon e^{i K \xi} e^{i\left(K v_{g l}-\Omega\right) t} .
$$

When $\epsilon$ is zero, Eq. (8) is integrable along the steps we have followed above. An interesting point about that case is that after our coordinate transformations, the equation is converted into one independent of the spatial variable $x$. With this in mind we see that if $a_{1}(t)$ is a possible solution, so is $a_{p}(t-\lambda \xi)=a_{p}\left(t-\lambda\left(x-v_{g l} t\right)\right)$ (with arbitrary $\lambda)$, for example, which means that from a purely temporal solution, we can generate traveling-wave solutions that propagate with velocities $v_{g l}+1 / \lambda$. For small values of $v_{g l}$, the usual case, one notices that for $\lambda$ smaller or larger than 1, we have superluminous and subluminous propagation, respectively.

In the case where $\epsilon \neq 0$, things become more complicated. Our equation becomes a nonintegrable one (the factor $H$ is no longer conserved) and a numerical analysis is necessary. We did that for the case of a strongly modulated train with $\epsilon=1$ and $\xi=0$, with a value of $\delta$ below the critical one. We chose $\epsilon=1$ as a typical value because smaller values would imply, in much longer time scales, changes in amplitudes of the plasma waves; again, this would be an adiabatic process not compatible with beam loading.

In Fig. 2 we compare the solutions for the case $\epsilon=0$, $\delta=0$ (and a pump amplitude $\sqrt{3 / 2}$ rather than 1 in order to assure the laser energy of the perturbed and unperturbed systems to be exactly the same) with the one defined by $\epsilon=0, \delta=-3$. Much in accordance with Fig. 1, one sees a dramatic reduction in the plasma wave amplitude; the large amplitude modes are not accessible from noise-level initial conditions.

In Fig. 3 we compare the solution $\epsilon=0, \delta=0$, which is thought to be the most practical one in the case of homogeneous pumps [8], with the one defined by $\epsilon=1$, $\delta=-3.0, \xi=0$. In this situation, where we have taken the frequency of the laser modulations to be in resonance with the nonlinear period of the unperturbed electrostatic mode, one sees an appreciable amplification of the plasma wave in few periods of the nonlinear frequency.

Although for this kind of intense perturbation it is difficult to speak in terms of the "nonlinear frequency of the unperturbed systems," one can still think of it as an extrapolation of the case of small perturbations. In this regard, what is happening is that, as the system resonantly absorbs energy from the perturbed pumps, it passes from a state of small amplitudes to another with large amplitudes, because the above-mentioned potential barrier $[9,10]$ that separates those two classes of roots is destroyed during the modulation period. That can be verified if one turns the modulation off when the ampli-

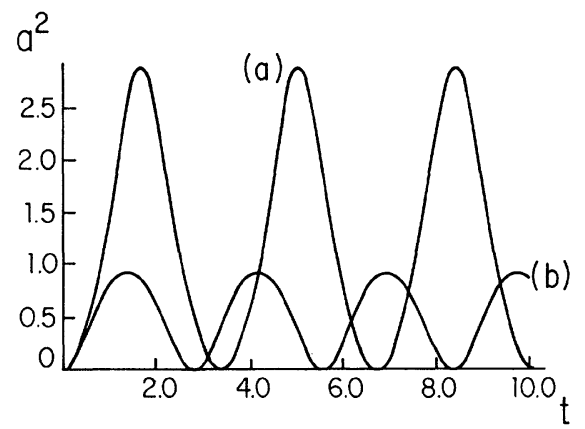

FIG. 2. A comparison of oscillations induced by homogeneous pumps, for plasma waves that grow from the noise level; curve $(a) \delta=-3$ and curve $(b) \delta=0$. 


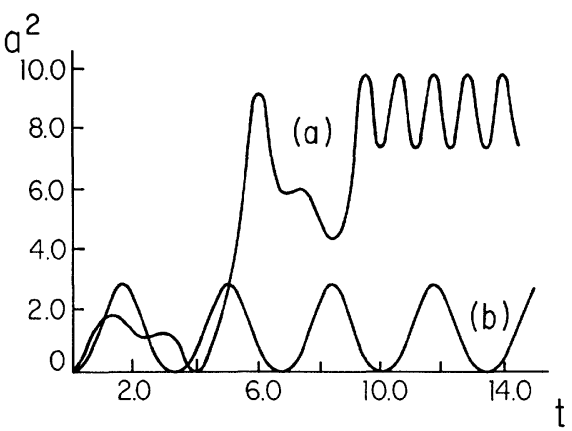

FIG. 3. Oscillations with modulated [curve $(a)$ ] and homogeneous [curve $(b)$ ] pumps. For curve $(a) \epsilon=1, \delta=-3$; for curve $(b), \epsilon=0, \delta=0$. The oscillatory behavior of $a$ occurs after the modulation is turned off.

tude attains the maximum peak. Then, the final oscillatory behavior of Fig. 3 (after turning off) would correspond to oscillations in the allowed region of Fig. 4, which is constructed with $H$ calculated at the peak of Fig. 3 and where indeed no "barrier" separating small and large roots is seen. We did not get any relevant amplification for values of $\delta$ below -3.2 , because in this case the potential barrier is too high to be completely destroyed. However, we would like to point out that if one compares Figs. 1 and 4 it is possible to see that our final state could be considered as equivalent to the initial one with $\delta \approx-4.65$. No amplification as appreciable as the last one was noticed in a few periods when we took the condition of optimal dephasing $K \xi=\pi, 3 \pi, \ldots$, for in this case there is destructive interference between the perturbation and the original oscillations. Interestingly, from this last point we conclude that the amplification of the electrostatic field will be highly varying and periodic along the space and time; if one wishes maximum efficiency, one must keep following pulses defined by $\cos K \xi=0$ with velocity $v_{g l}$.

Although the type of modulated laser beam we are employing in this paper may be quite adequate to describe modulations artificially produced in the laboratory, it is not the most appropriate one to describe MI, because in this last situation what we have is not only one harmonic mode being excited but an entire wave packet with several harmonic components [6]. So, from this present perspective one may visualize MI as a damaging process that can disrupt the sharpness involved in the construction of our resonance conditions, therefore limiting the efficiency of the whole process; indeed, the size of the electrostatic beat wave would be physically limited by the time over which MI develops. This appears not to be quite so severe a restriction, since in general the MI time scale of the laser field is longer than the "build up" time for homoge-

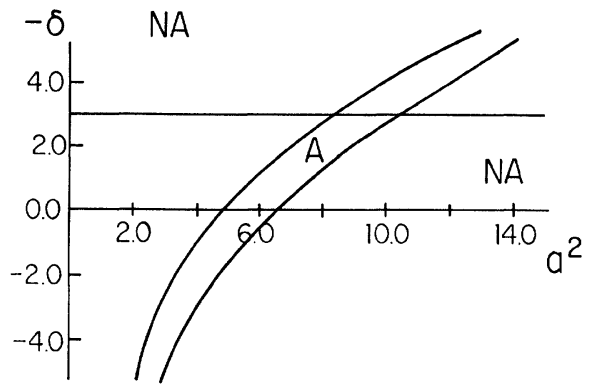

FIG. 4. State of the system (in terms of $H$ ) after the modulation is turned off. The new region of oscillation is shown.

neous systems [11], which is about the same as the one for our modulated case (see Fig. 3). Another important observation to be made is that the electrostatic beat wave is also modulationally unstable [8]. This kind of instability can also reduce the efficiency of the process by affecting the nonlinear resonances via frequency shifts, but if one considers very weakly dispersive systems [an approximation already used to discard the second derivatives of our basic equation, Eq. (1)], its characteristic time scales are longer than the one we are interested in. We should still point out that MI may be an important factor in the particle-acceleration time scales [6], but again, in such a stage the plasma wave has already attained its maximum value and no concern about the interference between artificial and unstable modulations is really necessary.

To summarize, it is seen that modulations of the laser beams of the beat-wave accelerator scheme could induce the generated plasma wave to be intensified to large values that are physically inaccessible when it grows from the noise level in completely homogeneous systems. This kind of amplification does not require any extra energy for the laser fields and originates as the plasma wave climbs its own effective potential well, with a subsequent destruction of the separatrix $[9,10]$ that separates the small and large roots representing maxima of its amplitude, a fact made possible due to the presence of resonances and resonance overlaps involving the nonlinear frequency of the plasma-wave envelope and the modulation frequency of the laser pumps in the case of optimal phase, $K \xi=0,2 \pi, \ldots$ Calculations with more refined models (as, for example, with the more realistic situations of localized electromagnetic pulses) will be reported elsewhere.

\section{ACKNOWLEDGMENTS}

I would like to thank Dr. M. C. Barbosa for a critical reading of the manuscript. This work was supported by Conselho Nacional de Desenvolvimento Científico e Tecnológico (CNPq) and Financiadora de Estudos e Projetos (FINEP), Brazil.
[1] T. Tajima and J. M. Dawson, Phys. Rev. Lett. 43, 267 (1979).

[2] M. N. Rosenbluth and C. S. Liu, Phys. Rev. Lett. 29,
$701(1972)$.

[3] C. M. Tang, P. Sprangle, and R. N. Sudan, Phys. Fluids 28, 1974 (1985) 
[4] R. J. Noble, Phys. Rev. A 32, 460, (1985).

[5] C. E. Max, J. Arons, and A. B. Langdon, Phys. Rev. Lett. 33, 209 (1974).

[6] C. J. McKinstrie and R. Bingham, Phys. Fluids B 1, 230 (1989).

[7] A. C.-L. Chian and C. F. Kennel, Astrophys. Space Sci. 97, 9 (1983).
[8] C. J. McKinstrie and D. W. Forslund, Phys. Fluids 30, 904 (1987).

[9] B. V. Chirikov, Phys. Rep. 52, 263 (1979).

[10] A. J. Lichtenberg and M. A. Lieberman, Regular and Stochastic Motion (Springer-Verlag, Berlin, 1983).

[11] P. Gibbon, Phys. Fluids B 2, 2196 (1990). 